\title{
Dynamics of vortices and knots in Faddeev's model
}

\author{
Jarmo Hietarinta*1, Juha Jäykkä ${ }^{1}$ and Petri Salo ${ }^{2}$ \\ ${ }^{1}$ Department of Physics, University of Turku, FIN-20014 Turku, Finland \\ ${ }^{2}$ Laboratory of Physics, Helsinki University of Technology, FIN-02015 HUT, Finland \\ E-mail: hictarin@utu. fij,juoljoautu.fi, ipetri.salo@hut. fij
}

Abstract: We review work on the minimum energy configurations of the FaddeevSkyrme model. The model contains a unit vector-field defined in the three dimensional space and pointing to a fixed direction at spatial infinity, the field configurations are partially characterized by the Hopf charge. This model, proposed by Faddeev in 1975, allows for localized and stable finite-energy configurations with nonzero Hopf charge. We discuss the deformation process of (linked) unknots in which a given initial vector-field evolves to the minimum energy configuration under dissipative dynamics.

\section{Knots in physics}

Knots have occupied physicists for more than a century. Lord Kelvin (1869) was perhaps the first to use knots in explaining physical phenomena: he proposed to consider atoms as knotted tubes of ether. Classification of knots would then give classification of atoms. This proposal did not work in physics, but it gave an impetus in the mathematical study of knots.

When we consider physical knots the first questions is: What kind of material can form knots? There are simple physical examples, e.g., rope or DNA, both of which are modeled by localized material in empty space.

In this paper we review the work of [i]1, , $\overline{2}]$, in which the object (line) that forms knots is the vortex core. In particular the vortex "material" is defined everywhere, and its particular value defines the core. For example, in the case of a tornado, hurricane, or maelstrom the velocity vector is defined everywhere and the vortex is associated to a special point in its distribution (e.g., the eye of hurricane). However, a velocity vector can decrease continuously to zero, and therefore there cannot be any topological conservation laws and vortices can disappear. In this paper we only consider permanent vortices, whose existence is guaranteed by a topological conservation law.

\footnotetext{
${ }^{*}$ Speaker.
} 


\section{Topology}

A typical example of a topological conservation law on the plane is the number of times a closed path goes around a fixed point. This number is conserved under all deformations in which the path does not cross the chosen fixed point. More precisely we should consider a closed curve on a punctured plane $f: S^{1} \rightarrow \mathbb{R}^{2} \backslash\{(0,0)\} \approx S^{1}$, and topologically such maps can be classified into homotopy classes according to the winding number $\pi_{1}\left(S^{1}\right)=\mathbb{Z}$.

What kind of topological conservation laws can we have in $\mathbb{R}^{3}$ ?

To each point in the space $\mathbb{R}^{3}$ we associate a $3 \mathrm{D}$-unit vector $\mathbf{n}$. These $3 \mathrm{D}$-unit vectors can be represented by points on the surface of the sphere $S^{2}$. We assume the vectors point to the same direction at spatial infinity and therefore the spatial infinity can be contracted to a point, i.e., $R^{3} \approx S^{3}$. Thus, the unit vector provides us the map $\mathbf{n}: S^{3} \rightarrow S^{2}$. Such maps can be characterized topogically by a Hopf charge, i.e., by the homotopy class $\pi_{3}\left(S^{2}\right)=\mathbb{Z}$.

The simplest unit vector-field with Hopf-charge $Q=-1$ is the following (Whitehead 1947)

$$
\begin{aligned}
& n_{1}=\frac{4\left(2 x z-y\left(x^{2}+y^{2}+z^{2}-1\right)\right)}{\left(1+x^{2}+y^{2}+z^{2}\right)^{2}}, \\
& n_{2}=\frac{4\left(2 y z+x\left(x^{2}+y^{2}+z^{2}-1\right)\right)}{\left(1+x^{2}+y^{2}+z^{2}\right)^{2}}, \\
& n_{3}=1-\frac{8\left(x^{2}+y^{2}\right)}{\left(1+x^{2}+y^{2}+z^{2}\right)^{2}} .
\end{aligned}
$$

Note that $\mathbf{n} \rightarrow(0,0,1)$ as $x^{2}+y^{2}+z^{2} \rightarrow \infty$, and the preimage of $\mathbf{n}=(0,0,-1)$ is the ring $z=0, x^{2}+y^{2}=1$, which forms the vortex core.

Later we will discuss how the Hopf charge is obtained using knot-theoretical concepts. Here we mention how it can be ontained from $\mathbf{n}$ using calculus: Given the unit vector field $\mathbf{n}: \mathbb{R}^{3} \rightarrow S^{2}$ we define $F_{i j}=\epsilon_{a b c} n^{a} \partial_{i} n^{b} \partial_{j} n^{c}$. Given $F_{i j}$ we must next construct $A_{j}$ so that $F_{i j}=\partial_{i} A_{j}-\partial_{j} A_{i}$, clearly $A_{i}$ can only be defined up to a gauge $A_{i} \rightarrow A_{i}+\partial_{i} \Phi$. A convenient gauge is $A_{3}=0$, then from $F_{13}=-\partial_{3} A_{1}$ we can integrate $A_{1}$, and $A_{2}$ from $F_{23}=-\partial_{3} A_{2}$, the integration constants are then determined from $F_{12}=\partial_{1} A_{2}-\partial_{2} A_{1}$. Finally we define

$$
Q=\frac{1}{16 \pi^{2}} \int \epsilon^{i j k} A_{i} F_{j k} d^{3} x
$$

This procedure can be easily implemented numerically.

\section{How to visualize vector fields?}

Before going into specific models and their vector-field solutions let us consider the problem of visualizing the vector field. Clearly we cannot draw vectors at every point in space. Furthermore, flow lines do not make sense because of global gauge invariance, in fact our vectors could live in another space, e.g., the internal spin space.

As described above, vectors $\mathbf{n}$ can be represented by points on the sphere. We have already one fixed direction, $\mathbf{n}$ at infinity, which we define as the north pole, i.e., $\mathbf{n}_{\infty}=(0,0,1)$. 

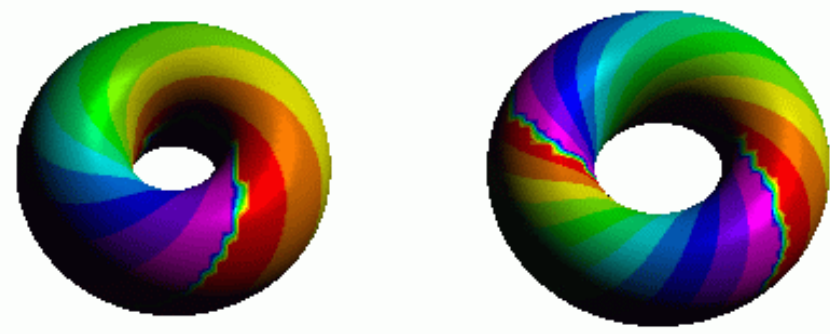

Figure 1: Isosurface $n_{3}=0$ for charge -1 and -2 unknots [i].

All other vectors can be characterized by the latitude and longitude of the corresponding point on the sphere. Latitude is invariant under global rotations (it is characterized by the value of $\mathbf{n}_{\infty} \cdot \mathbf{n}$ ), and therefore it is informative to plot equilatitude surfaces. Longitudes are represented by colors on the equilatitude surface, which is natural because colors are associated with the "color circle". (Under a global gauge rotation only colors change). The $n_{3}=0$ isosurface shown in Figure $i_{-1}^{1}$ is just one of the isosurfaces we can draw, and inside is the core line, where $n_{3}=-1$. Alternatively we could draw preimage-curves of various n values. For example, since the coloring in Figure $\underline{1}_{1}^{\overline{1}}$ is by latitude the color boundaries are preimages of various point on the equator. The order of colors and the handedness of twist on the torus determine Hopf charge through their association to linking numbers of the preimages, this will be discussed further in Section $\underline{\underline{6}}$.

\section{Faddeev's model and its numerical investigation}

In addition to topology we need physics. In 1975 Faddeev [i3i] proposed the following Lagrangian (energy) for the unit vector field $\mathbf{n}$ :

$$
E=\int\left[\left(\partial_{\mu} \mathbf{n}\right)^{2}+g F_{\mu \nu}^{2}\right] d^{3} x, \quad F_{\mu \nu}:=\epsilon_{a b c} n^{a} \partial_{\mu} n^{b} \partial_{\nu} n^{c} .
$$

Note that under the scaling $\mathbf{n}(r) \rightarrow \mathbf{n}(\lambda r)$ the integrated kinetic term scales as $\lambda$ and the integrated $F^{2}$ term as $\lambda^{-1}$, and therefore in this model nontrivial configurations will attain some fixed size determined by the dimensional coupling constant $g$ (Virial theorem).

In 1979 Vakulenko and Kapitanskii [i] for the energy of this model, $E \geq c|Q|^{\frac{3^{4}}{4}}$, where c is some constant and $Q$ the Hopf charge. Note the $3 / 4$ power in the $Q$ dependence. An improved estimate for the constant $c$ was

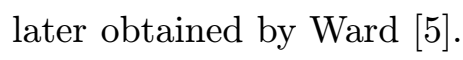

Some other field theoretical models having configurations with nonzero Hopf charge have also been proposed, these include

- de Vega (1978), Higgs-models (Abelian and SU(2)) [ị̂ㅁ]

- Nicole (1978), $\mathcal{L}=-\left[-\frac{1}{4}\left(\partial_{\mu} n^{a}\right)^{2}\right]^{3 / 2}[i \bar{i}$,

- Kundu and Rubakov (1982), $S^{2}$ nonlinear $\sigma$-model [i⿱亠凶禸] $]$,

- Aratyn, Ferreira, Zimerman (1999), $\mathcal{L}=-\left[F_{\mu \nu}^{2}\right]^{3 / 4}[\underline{\overline{9}}]$. 


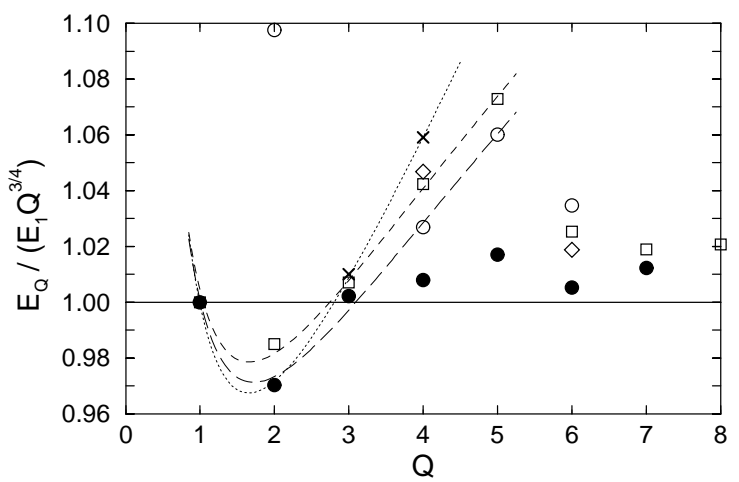

Figure 2: Energy values for various configurations [2]. Filled circles give the best result for a given Hopf charge; open squares are from [1] Note the scaling by $E_{(|Q|=1)}|Q|^{3 / 4}$.

The first numerical studies of Faddeev's model were done by Gladikowski and Hellmund [1 [10 1 and 2 within the cylindrical ansatz; they also considered other choices for the energy functional. Then Faddeev and Niemi [i] 110 in presented results on charges 1 and 2, still within cylindrical ansatz, but they also presented conjectures on the trefoil knots, which generated new activity in this field. Soon after Battye and Sutcliffe [i] 1 2-1] $]$ presented comprehensive results for charges 1-8. Their initial states were torus type unknots, and for higher Hopf charges the results show interesting deformations, including deformation to the trefoil knot for charge 7. Hietarinta and Salo [i] considered several linked initial configurations, and they also saw deformation into the trefoil knot for charge 7. A further study [2] revealed more details on the lowest energy states, resulting with a very good agreement with the VK bound, as can be seen in Figure

\section{Linked unknots}

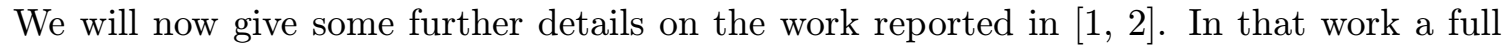

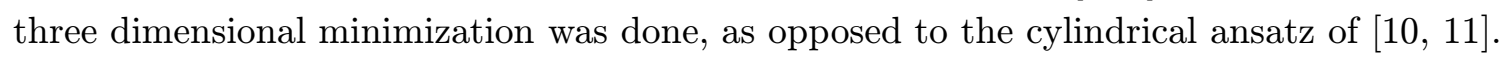

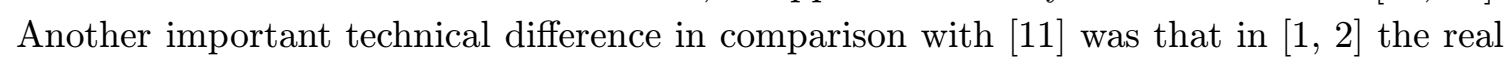
unit vector field $\mathbf{n}$ was used, rather than the complex scalar field $W=\left(n_{1}+i n_{2}\right) /\left(1+n_{3}\right)$, which in principle carries the same information. This was done because the singularities in $W$ (i.e., points where $n_{3}=-1$ ) are hard to handle numerically. Of course when one uses $\mathbf{n}$ one has to make sure that $|\mathbf{n}(x, y, z)|=1$ at all times, this was guaranteed by scaling back to $|\mathbf{n}|=1$ after each iteration step (15.15).

In the numerical implementation we decided to use a discretization that allows straightforward parallelization. The system was discretized on a regular cubic lattice, size typically $240^{3}$. In discretizing the Lagrangian ( $\left.\overline{4}^{4} \cdot \bar{l}^{\prime}\right)$ we discretized $\partial_{i} \mathbf{n}$ on links, $F_{i j}$ on plaquettes. Using more lattice points to discretize a derivative would perhaps be more accurate, but with our choice the code parallelization was simpler since only one boundary layer had to be exchanged between processors. From the discretized Lagrangian we computed the gradient $\nabla_{\mathbf{n}(\mathbf{r})} L$ symbolically and the minimum energy configuration was then found using dissipative dynamics

$$
\mathbf{n}_{\text {new }}(\mathbf{r})=\mathbf{n}_{\text {old }}(\mathbf{r})-\delta \nabla_{\mathbf{n}(\mathbf{r})} L, \forall \mathbf{r} .
$$

Program parallelized well, and we have used various parallel machines, including Cray T3E, SGI Origin 2000, IBM RS/6000 SP Cluster.

One novel idea in [1]1, knots, namely linked unknots. Such initial states were constructed by taking two unknots, 

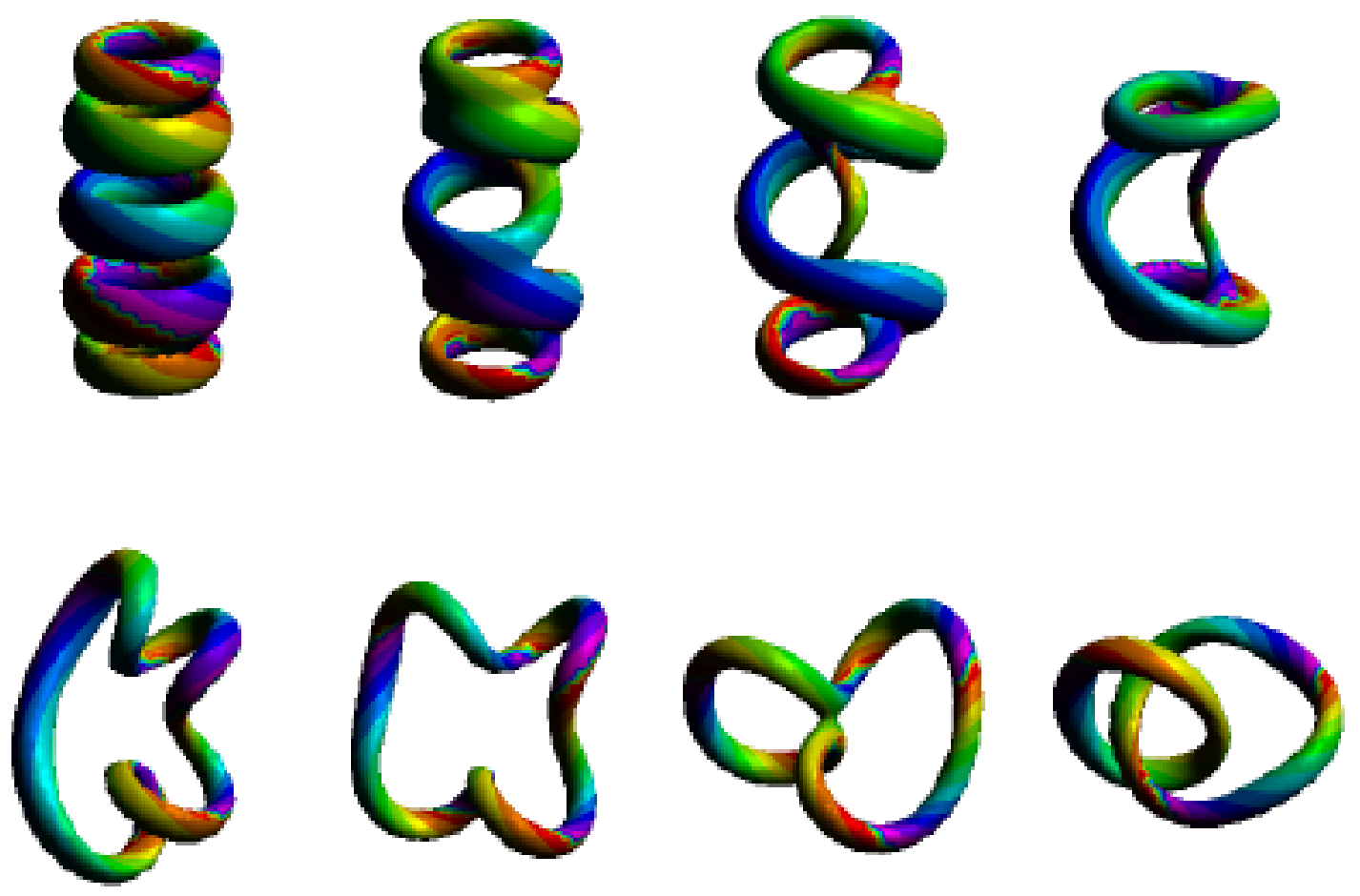

Figure 3: Snapshots of the deformation of a $(1,5)$ unknot (upper left) into a $1+2+2$ link (lower right).

cutting each into 4 parts, and then recombining them by filling some sections with straight tubes, others with the vacuum (cf. Fig. 2 in [i] $\left.]_{1}^{1}\right)$. The total Hopf charge of such a linked combination is the sum of charges of individual unknots plus their linking number. The linking number depends on the relative direction associated with the unknots, this will be discussed more in Section :

In [i] various ways of linking charge $|Q|=1,2$ unknots were considered. Perhaps the most interesting results was the sequence by which a linked configuration $-4-5+2$ relaxed

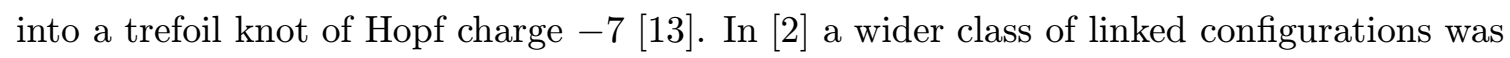
considered and as a result even better minimum states were found. Unknots with torusshaped isosurfaces can have color boundaries (preimages of points on the equator) that rotate $n$ times around the small circle and $m$ times around the large one, such configurations

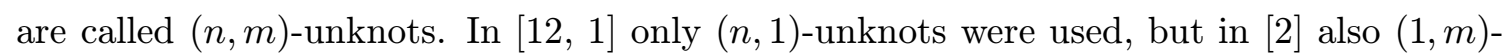
unknots were included. The deformation of $(1,5) \rightarrow 1+2+2$ is illustrated in Figure best new results turned out to improve agreement with the VK bound, as is illustrated in Figure $\overline{2}$.

\section{Knot theory}

The proper knot-theoretical setting for the analysis of knots associated to unit vector fields is to use framed links. This is because it is not sufficient to know the location of the vortex 


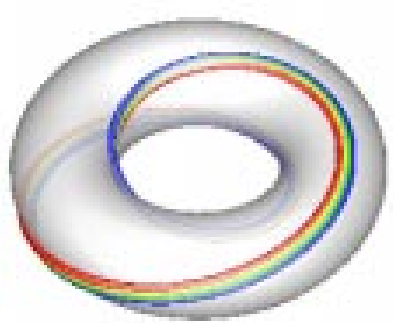

Figure 4: $(1,2)$ torus.

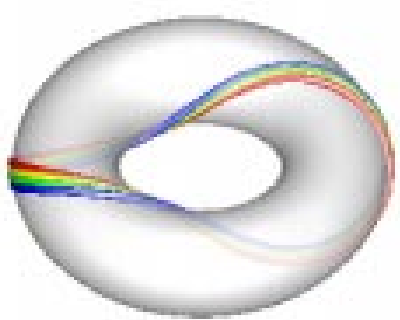

Figure 5: $(2,1)$ torus.

core (=the preimage of the south pole of the map $\mathbf{n}: \mathbb{R}^{3} \approx S^{3} \rightarrow S^{2}$ ), but also the twisting around the core, and the framing attached to a curve adds this needed local information. In order to visualize framed links we use directed ribbons, which are preimages of line segments. We can use equilatitude line segments, then increasing latitude and longitude give two directions and their cross product gives the ribbon direction. For practical reasons we usually replace the ribbon by 4 nearby colored lines, Figures 雷 and define

The Hopf charge can be computed from the directed preimage-lines as follows [i] $\left.{ }^{[} \overline{4}\right]$ : We

twist $=$ twist along the ribbon axis, i.e., linking number of the ribbon core with one of the (adjacent) ribbon boundaries,

writhe $=$ signed crossing number of the ribbon core with itself,

where linking number $=\frac{1}{2}$ (sum of signed crossings).

With these definitions we can calculate the Hopf charge using either of the two formulae

a) twist + writhe,

b) linking number of the two ribbon boundaries.

One can also show that computing Hopf charge from $\left(\overline{2} . \overline{1} \overline{1}_{1}^{1}\right)$ gives the same number as the

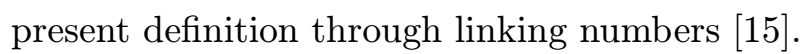

The sign convention used for the crossing of two directed lines is given on the leftmost part of Figure $\underline{6}$. If one takes the charge -1 torus unknot illustrated in Figure ${ }_{1}^{\overline{1}}$, then the

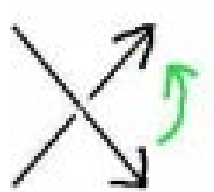

$+1$

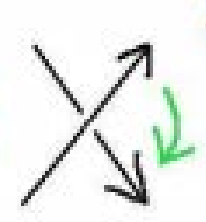

$-1$
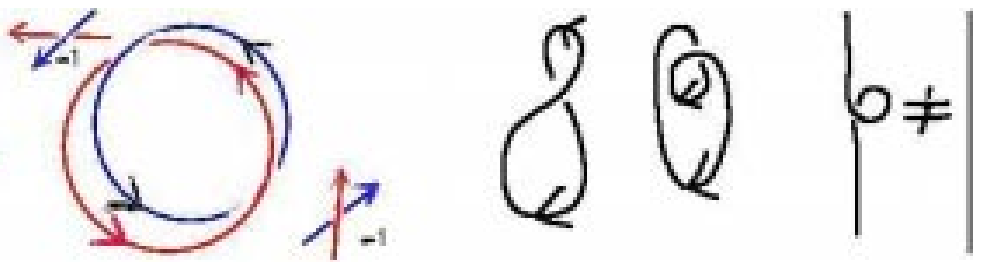

Figure 6: On the left: sign conventions for crossings, and application to the charge 1 unknot of Figure $\overline{1}_{\mathrm{r}}$ On the right how ribbons look when turned vertically, twist becomes a crossing, and therefore the Reidemeister move I is not allowed. 


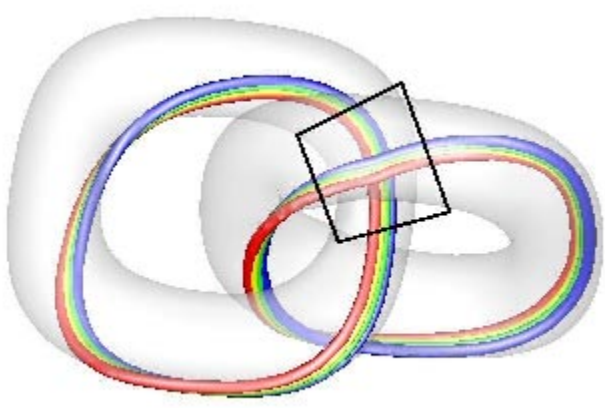

Figure 7: Initial state: two linked ribbons, with red lines close to each other.

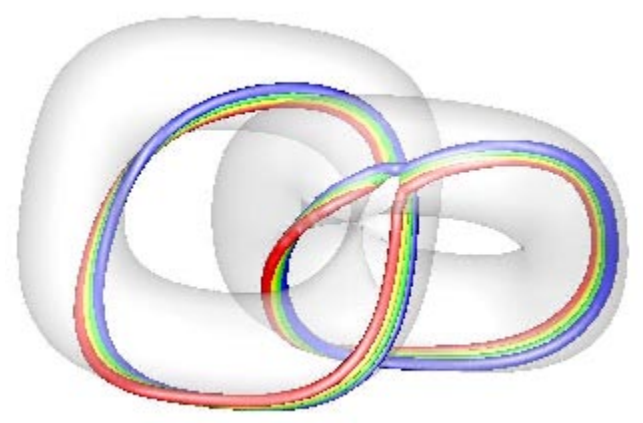

Figure 9: Also yellow and green lines have changed, blue still forms two circles.

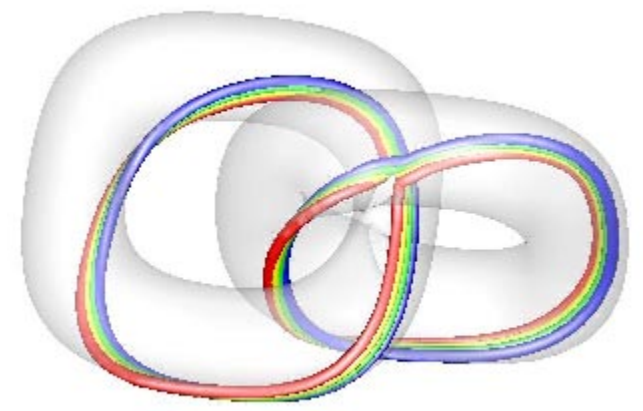

Figure 8: Red lines have touched and redistributed at intersection, now forming one big circle.

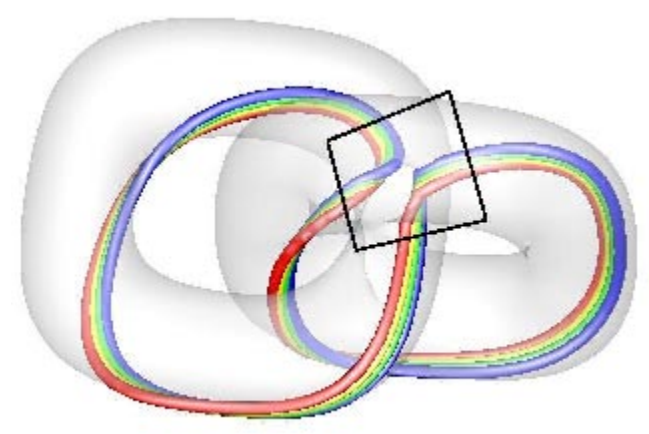

Figure 10: The ribbon now forms one big circle.

red and blue lines in Figure $\overline{6}$, correspond to any two consecutive color boundaries. The Hopf charge is obtained by counting the "linking number of the two ribbon boundaries", ribbon in this case being a band of solid color. On the right of Figure $\overline{6}_{6}^{\prime}$ we have shown how the ribbon looks when it has been turned vertically and is viewed from above (as is usually done with knot diagrams): a twist in the ribbon becomes a crossing. Note that when considering equivalence of framed diagrams the type I Reidemeister move is not allowed, it would remove a twist from the ribbon.

Returning to Figures 㓙 and we can see that there are two ways to obtain such configurations, we can go twice around small vs. large circle. In Figure $\underline{4}_{i}^{\prime}$ we have twist $=-1$, writhe $=-1$, and in Figure $\overline{\bar{s}}_{1}$ twist $=-2$, writhe $=0$, but in both cases we have boundary linking number $=-2$.

\section{Ribbon deformations}

One of the most fascinating things seen in the time evolution of the energy minimization [i] $\left.{ }^{1} \overline{3}_{1}\right]$ is the way the configurations deform into each other, for example how the trefoil is formed. The topological data in the vector field can be coded into framed links or ribbons, 


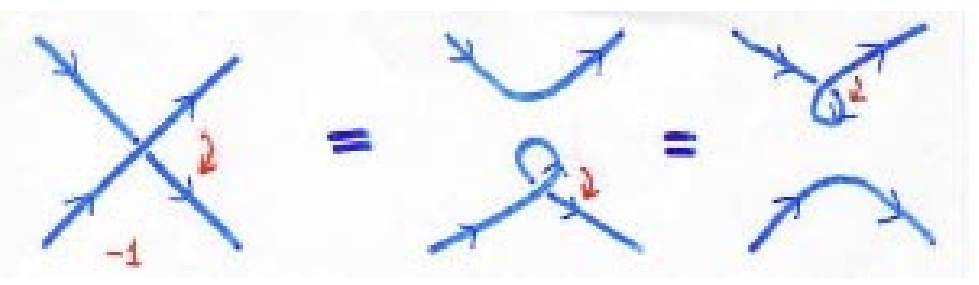

Figure 11: Possible ribbon deformations.

and knot deformations correspond to ribbon deformations in which the Hopf charge is conserved.

Ribbon deformation can be seen if we look the same configuration at different latitudes (see Figures 1 uous deformation and therefore it can happen only when two ribbons approach each other with edges of the same color. When these same color lines touch they can redistribute and when this is extended to the whole ribbon the process is as in Figures knot diagrams this means that we can have deformations like in Figure

\section{Physically relevant extensions}

Faddeev's model is the basic model that displays a rich phenomenology. One important question is whether it is relevant in the description of observed physical processes. The answer seems to be positive, and Faddeev's model seems to be hidden in many more physical models, some of the phenomena already discussed in this context include: electrically

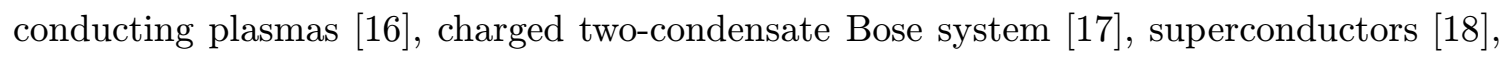
and Weinberg-Salam model [i] $\left.{ }_{1}^{1} \overline{9}\right]$.

For example, Babaev et al [i] $\left.\bar{i}_{1}\right]$ have proposed that Faddeev's model resides inside the Gross-Pitaevskii model of two electromagnetically coupled oppositely charged Bose condensates: After a suitable change of variables one obtains an energy functional containing Faddeev's model, coupled to several other fields. It is still an open question whether one can keep the topological structure with these extra fields; they sometimes appear multiplicatively and if they vanish the scaling properties change.

Vortices in superfluid ${ }^{3} \mathrm{He}$ present another interesting application, especially in the CUV-phase [2] $\left.\bar{Q}^{\prime}\right]$.

\section{Conclusions}

Our numerical results show that in Faddeev's model a given initial configuration of linked unknots can deform to a final, minimum energy configuration through a very complicated process. Each configuration can be characterized with a Hopf charge which is conserved during the deformation. The deformations can be understood in terms of framed ribbons and their allowed deformations, as described in knot theory. There is now increasing evidence that Faddeev's model and its extensions have a variety of different applications in various fields of physics. 


\section{Acknowledgments}

Supported in part by the Academy of Finland under grant 47188 (JH and JJ) and through its Center of Excellence program (PS). We also acknowledge the generous allocations of computer resources from CSC - Scientific Computing Ltd., Espoo, Finland. We would also like to thank L. Faddeev, L. Ferreira, C. Cronström and E. Haviola for discussions.

\section{References}

[1] J. Hietarinta and P. Salo: "Faddeev-Hopf knots: dynamics of linked unknots", 'P (104)

[2] J. Hietarinta and P. Salo: "Ground state in the Faddeev-Skyrme model", (2000) $08 \overline{1} \overline{1} \overline{1} \overline{1}$.

[3] L. Faddeev, IAS Print 75-QS70 (1975); L. Faddeev, "Einstein and Several Contemporary Tendencies in the Theory of Elementary Particles", in "Relativity, Quanta, and Cosmology, Vol.1", M. Pantaleo and F. De Finis, eds., pp. 247-266 (1979), reprinted in L. Faddeev, "40 years in mathematical physics", pp. 441-461 (World Scientific, 1995).

[4] A.F. Vakulenko and L.V. Kapitanskii, Sov. Phys. Dokl. 24 (1979) 433.

[5] R S Ward, "Hopf solitons on S(3) and R(3)," Nonlinearity 12 (1999) 241.

[6] H. J. de Vega, "Closed vortices and the Hopf index in classical field theory," 'Phys. Rev. D 18" - - -

[7] D. A. Nicole, "Solitons with non-vanishing Hopf-index,"

[8] A Kundu and Yu P Rybakov, "Closed-vortex-type solitons with Hopf index", U. Phys. A - - -

[9] H. Aratyn, L. A. Ferreira, and A. H. Zimerman, "Toroidal solitons in 3+1 dimensional integrable theories," "Phys. Lett. B $\mathbf{4} \overline{\mathbf{5}} \mathbf{6}(1999) 162_{1}$, "Exact static soliton solutions of $3+1$ dimensional integrable theory with nonzero Hopf numbers," iPhys. Rev. Lett. 83. (1999) 1723.

[10] J. Gladikowski and M. Hellmund, "Static solitons with nonzero Hopf number", 'Ph -

[11] L. Faddeev and A. Niemi, iNature $\mathbf{3 8 7}(1997)$ 58.

[12] R.A. Battye and P.M. Sutcliffe, "Knots as Stable Soliton Solutions in a Three-Dimensional Classical Field Theory", "Phys. Rev. Lett. 81 (1998) 4798 .

[13] htttp://users.utu.fílhietarin/lknots/index.htm]'

[14] K. Murasugi, "Knot Theory \& its applications", Birkhäuser (1996).

[15] R. Bott and L. Tu, "Differential forms in algebraic topology" Springer (1982).

[16] L. D. Faddeev and A. J. Niemi, "Magnetic geometry and the confinement of electrically conducting plasmas," "Phys. Rev. Lett. 85 (2000) $34 \overline{1}$.

[17] E. Babaev, L. Faddeev and A. Niemi, "Hidden symmetry and duality in a charged two-condensate Bose system", "Phys. Rev. B 65 (2002) 100512i; Y. M. Cho, "Creation of Knots in Bose-Einstein Condensates," 'cond-mat/0112325: 
[18] E. Babaev, "Knotted solitons in triplet superconductors," 'P hys. Rev. Lett. 88 $(2002)$ 17700;" "Vortices carrying an arbitrary fraction of magnetic flux quantum in two-gap superconductors," 'Phys. Rev. Lett. $\mathbf{8 9}$ ' $(2002)$ 2067001'

[19] Y. M. Cho, "Knot solitons in Weinberg-Salam model," hep-th/01100̄70̄

[20] Ü. Parts, J. M. Karimäki, J. H. Koivuniemi, M. Krusius, V. M. H. Ruutu, E. V. Thuneberg, and G. E. Volovik, "Phase Diagram of Vortices in Superfluid ${ }^{3} \mathrm{He}-\mathrm{A}$ ", Phys. Rev. Lett. 75 "

(1995) 3320 ;i; O. Lounasmaa and E. Thuneberg, "Vortices in rotationg superfluid ${ }^{3} \mathrm{He}$ ", Proc.' Nat. Acad. Sci. $\mathbf{9} \mathbf{6}(\overline{9} 9 \overline{9}) \overline{7} \overline{6} \overline{0}$. 